\title{
Los peligros de la investigación multidisciplinaria en estudios fronterizos. Un dilema profesional para los académicos "generalistas"
}

\author{
Ellwyn R. Stoddard \\ Universidad de Texas en El Paso
}

\section{Resumen}

En este trabajo realizado a través de un ensayo biográfico, se comentan los peligros y obstáculos a que se enfrenta el académico cuando al abordar el problema del estudio de la frontera, pierde parte de su especialización para convertirse en académico multidisciplinario "generalista", esto como una situación inaplazable si desea realmente profundizarse en el conocimiento de los territorios fronterizos. Se seleccionaron algunas de las aportaciones y conceptos de los estudios fronterizos, de manera que se vislumbren y ordenen los problemas de los académicos en la multidisciplina. Se reordenaron los materiales mediante un esquema cronológico, para mostrar cómo algunas de las aportaciones a los estudios fronterizos han surgido debido a las múltiples influencias multidisciplinarias.

Palabras clave: investigación multidisciplinaria, estudios fronterizos, académicos generalistas, estereotipos étnicos.

\begin{abstract}
This work, which is performed through a biographical essay, comments on the dangers and obstacles that academics find when they try to research on border issues. They god part of their specialty is lost when they become a "generalist multidisciplinary academic". If one really pretend to deepen on the knowledge of border territories, it becomes a pressure problem to discover and to order the academics problems on multidiscipline. This material was ordered in chronological order to be able to show how the discoverings on border researches obtained have appeared due to the multi-disciplinary influence.
\end{abstract}

Keywords: multi-disciplinary research, border studies, generalist academics, ethnical stereotypes. 


\title{
LOS PELIGROS DE LA INVESTIGACION MULTIDISCIPLINARIA EN ESTUDIOS FRONTERIZOS. UN DILEMA PROFESIONAL. PARA LOS ACADEMICOS "GENERALISTAS"?
}

\author{
Dr. Ellwyn R. Stoddard \\ Investigador del Depto, de Sociología y Antropología \\ de la Universidad de Tixas en El Paso.
}

Este ensayo examina los obstáculos y peligros que le aguardan al académico cuando se convierte en "generalista", en escolar multidisciplinario en el mundo presente de las computadoras electrónicas y la especialización. Al contemplar las variaciones que se pueden utilizar en este proyecto, seleccioné algunas de las aportaciones y conceptos de los estudios fronterizos, de manera que se vislumbren y ordenen los problemas de los acadénicos en la multidisciplina. Aunque la información fue cubierta, el ensayo fue una "revoltura"; asi que reordené los materiales en un esquema cronológico, mostrando cómo algunas (pero no todas) aportaciones a los estudios fronterizos han surgido đebido a las múltiples influencias multidisciplinarias. Este ensayo fue tan complejo que faltó continuidad al describir y analizar el problema central.

Así, en lugar del potencial de crítica negativa, intenté usar una narrativa del tipo "historia personal". Esta estructura refleja más acertadamente los esfuerzos y peligros que he observado o experimentado en mis intentos de convertirme en conocedor de alrededor de una docena de disciplinas, para actuar como centro de información y/o catalizador de mis colegas académicos fronterizos. Este estudio de caso está dirigido a investigadores en potencía, orientados a estudios fronterizos; los jóvenes académicos que se hallan en la encrucijada y pudieran ser atraídos por ideas románticas concernientes a la colaboración multidisciplinaria. Este trabajo pretende ponerlus al tanto de los peligros de su carrera y de las estructuras profesionales que les acompañarán en sus decísiones al seguir el camino del conocimiento generalizado, y también para mostrar las satisfacciones personales y aportaciones profesionales que resultarian de ahí en adelante.

\section{ROMANTICISMO: UN OBSTACULO INICIAL.}

La socialización temprana le da a cada persona un conocimiento de su mundo y las experiencias subsecuentes modifican esos puntos de vista. Cuando los primeros años de in fancia se viven en circunstancias restrictivas y carentes de estímulos externos, el aprendizaje subsecuente es bien recibido, como agua que se le da al sediento, y se consume sin mucha absorción inicial. En mi caso, mis conocimientos de otras culturas, religiones, tierras y reacciones complementarias, ampliaron mi conocimiento y a la vez me insensibilizaron

'Ponencia presentada en la Conferencia de Estudios Latínoamericanos de la Costa del Pacífico en Tijuana, B.C. México (Octubre 15, 1982). 
ante las diferencias entre personas de amplias categorías culturales. La incongruencia entre las categorías de las personas y sub-grupos o culturas, empezzron a edad temprana y continuaron durante los estudios de mi carrera.

Durante los primeros años de la Gran Depresión, mi padre perdió su rancho árido del desierto de Utah y, afortunadamente, me ví forzado a reunciar al nivel rural de pobreza para ir a vivir a la ciudad con mi abuelo. Ahí recibí una buena educación formal, incluyendo parte de la secundaria, y los desafortunados años de la 11 Guerra Mundial, cuando abandoné la escuela para ingresar al servicio militar. Esto me llevó a ampliar mis conocimientos por medio de los viajes y relaciones personales, más allá de los conocimientos de mis primeros años de cómoda protección familiar. La sencilla experiencia de visitar San Francisco, Nueva York, Baltimore y Washington, D.C., y visitar a los nativos de las islas del Pacífico, fue una experiencia emocionante. Cuando terminó mi servicio, me separé de mi familia por tres años y me fuí a la America Latina ${ }^{2}$, hablando una lengua totalmente diferente, pero viviendo con gente de posición económica muy similar a la de mi juventud.

Al regresar a los E.U.A. empecé a prepararme para una carrera en el servicio extranjero (como una cuestión romántica) con preferencia en América Latina, con trabajo académico en ciencias políticas, económicas y lenguas extranjeras. Tomé algunos cursos sobre América Latina-Historia/Economía/Sistemas Políticos y aprendí la "Leyenda Blanca", versión europea de la conquis$\mathrm{ta}^{3}$. Aunque los estereotipos románticos de los sistemas políticos que estudié fueron inconsistentes con los contactos directos y experiencias personales que disfruté con la gente latina, no estaba capacitado para cuestionar esta disparidad. Aprendí sobre la riqueza de las variaciones culturales en cada país; sin embargo, mi entrenamiento profesional se enfocó a los canales diplomáticos formales, por los cuales las tensiones internacionales pueden, eventualmente, aminorarse o resolverse.

Por mi calidad de reservista en tiempo de guerra (ROTC Program), me llamaron a servir durante el conflicto de Corea como oficial de artillería, reforzando mi etnocentrismo occidental. Mi subsecuente grado de maestría se con-

\footnotetext{
${ }^{2}$ De septiembre de 1946 a agosto de 1949, yo serví comó representante no asalariado de la iglesia misionera mormona, trabajando cerca de gente de habla española en varios lugares. Después de mi servicio en Texas, Brasil y Uruguay, yo pasé los treinta meses siguientes en varios lugares en Argentina. Es de este encuentro directo con gente placentera, que mi larga inclinación por el estudio de las culturas latinoamericanas cristalizć de una forma romántica, cambiándola gradualmente a una perspectiva científica.

${ }^{3} \mathrm{La}$ conquista del Nuevo Mundo puede ser tanto de una perspectiva de "Leyenda Blanca" o "Leyenda Negra". La "Leyenda Blanca" contempla la entrada de los españoles en América como una acción positiva, la cual trajo la civilización (cuitura europea) a las poblaciones nativas del norte y sur de América. William Prescott (1936), Lewis Hanke (1959) y los primeros trabajos de Charles Gibson, como la mayoría de los textos de historia del Sureste, refejarian el punto de vista de la "Leyenda Blanca". La parte de la "Leyenda Negra", describe a los conquistadores españoles como crueles, sádicos y tiranos y serán representados por los escritos de Fray Bartolomé de las Casas (1542) John Collies (1947) y Benjamín Keen (1969) aparte de otras.

Mis cambios cíclicos de un punto de vista a otro, similar a las experiencias de Gibson, han sido reconocidas (Stoddard, 1978: 34-35. Nota 5).
} 
centró tanto en el intenso problema político del día -el Mc Cartismo- 4 que me llevó casi dos años de mis estudios de doctorado en la Universidad del Estado de Michigan, antes de que mis estudios formales me exigieran resolver las diferencias entre las "culturas folklóricas" y los populares estereotipos nacionales de los países de América Latina. Trabajando en la investigación Carnegie Border Proyect, examiné artículos profesionales y monografías que utilizaban modelos de configuraciones culturales y valores de orientación ${ }^{5}$, en busca de sencillas determinantes étnicas de comportamiento. Aunque su valor analítico probó ser extremadamente limitado, su aceptación en la comunidad científica me cegó a la experiencia empírica de cultura heterogénea que había observado y vivido. Después de todo, lo que fue suficientemente bueno para algunos de mis respetados maestros y publicado por I. Lynn Smith (1956), dificilmente iba a ser cuestionado por un estudiante candidato al doctorado, menos aún a través de sus exhaustivos exámenes. Pero en mis momentos de especulación, me preguntaba porqué yo, de ascendencia galés-inglesa-irlandesa, cabía dentro de la "orientación de valores méxico-americano" en mi niñez, y sólo ahora, había llegado a ser un acreditado "anglo-americano". Al principio descarté esta incongruencia como una aberración única, pero cada vez más, en mis labores e investigación de campo empecé a darme cuenta que los valores mexico-americanos correspondían más a la gente pobre de cualquier grupo étnico mientras que la lista de los valores anglo americanos era un reflejo de los patrones culturales dominantes de la clase media. Pero pasaría más de una década, antes de que yo escribiera un libro sobre los mexico-americanos, intentando reemplazar aquellas nociones estereotipadas con información más objetiva y realista (Stoddard, 1973).

Al examinar críticamente muchos de estos errores de estereotipos étnicos, observaba cómo los nuevos conocimientos de la ciencia reemplazaban mis nociones románticas. La cada vez mayor objetividad hizo disminuir mi nos-

\footnotetext{
¿ Un senador de Minnesota, Eugene Mc. Carthy, postulado bajo la plataforma liberal del partido demócrata zara presidente; no es el de la "Era Mc Carthy" de que aquí se habla. El Senador de Wisconsin, José Mc. Carthy, rilizó la técnica de la "gran mentira" de acusar a otros de ser comunistas o simpatizantes de los comunistas 2 transformarla en una fuerza poderosa pero destructiva en la política americana de los primetos años de 1959 , la cual amenazó el proceso democrático. Ver el libro de Owen Lattimore's Ordeal by Slander, coma un sxudio de caso documentado de la acción del Mc Cartismo (1951).

SE modelo del valor de orientación, formulado por Florence Kluckhohn, en la tesis de maestría de 1937 diEencia las creencias polarizadas que representan la cultura española-americana (o mexicana-americana) y aglo-americana. Sus estudios de doctorado y post-doctorado en pueblos aislados y pequeños de Nuevo Mé. soo revelaron ciertos valores característicos que fueron conceptualizados con su contraparte urbana anglozericana y concibiéndolos como factores culturales étnicos. A partir de ahí, se dedujo que dichos factores zan la causa de los problemas entre ambos grupos sociales.

1 lista anglo-americana contenía los "valores preferidos", mientras que la hispana conten ía los menos funciozakes (véase Klukhohn y Stradtbeck. 1961, para una detallada elaboración).

Ias configuraciones culturales son matrices homogéneas de patrones culturales que, se pensaba, eran los resulados específicos de prácticas de educar al infante. Empezando con el trabajo de Ruth Benedict (1934), la zcela de personalidad y cultura se hizo muy popular como resultado del matrimonio entre conceptos psicoweicos (psiquiátricos) y datos etnográficos desarrollado entre el período 1940-1960. Véase el trabajo de Gorson Allport, Margaret Mead y otras durante ese tiempo, uno de los cuales estereotipó a los españoles americaaos de Nuevo México (Mead, 1955) en una forma errada y negativa.
} 
talgia y seguridad emocional. A medida que me adentraba en las investigaciones de las culturas de los indígenas de América Latina (y de los mexicoamericanos en los E.U.A.), empecé a ver la necesidad de una perspectiva y un panorama más amplios. Mi intención de ser multidisciplinario estaba fija. aún cuando mis conocimientos eran incompletos.

\section{LOS ESTUDIOS MULTIDISCIPLINARIOS DE LOS TERRITORIOS FRON- TERIZOS: FORMALIZACION DE UN CAMPO EMERGENTE.}

Habiendo ampliado mis conocimientos disciplinarios al incluir antropología, historia, demografía, cconomía agrícola, leyes y medicina ${ }^{6}$, me cambié a una universidad fronteriza - la universidad de Texas en El Paso (antes Texas Western College) - en 1965. En los meses siguientes empecé a buscar entre los colegas de la universidad, aquellos interesados en los fenómenos relacionados con la frontera. Todavía conservaba contacto con algunos colegas que habían trabajado en la Universidad del Estado de Michigan, en el proyecto fronterizo?

En 1968, una conferencia sobre urbanización fronteriza, me proporcionó la oportunidad de presentar un resumen de las investigaciones que se venían realizando o que se tenían terminadas en relación con tópicos de la frontera, entre académicos de muy diversas disciplinas. Fue entonces cuando me percaté de lo difícil que era para un académico sin publicaciones, lograr que se le aceptara tener una ponencia multidisciplinaria en las revistas concebidas como estrictamente disciplinarias. Finalmente recurrí a medios menos tradicionales, y me comprometí a elaborar una serie de artículos ocasionales para el Consorcio de Universidades de Estados-Fronterizos para América Latina (BSUCL.A), la cual agrupaba cuatro universidades fronterizas, incluyendo mi institución. Incluí mi resumen en el primer reporte eventual que se patrocinaba (Stoddard, 1970), teniendo en cuenta que 65 páginas, con un engargolado de plástico y un forro imprimido, no es una contribución "legítima" entre las reglas académicas. Aunque los dos folletos que edité hablando de la prostitución en la frontera y de los problemas de la droga tuvieron gran demanda, no había hecho gran cosa, o mejor dicho nada, por mi posición profesional. Observé entonces que los representantes de la BSUCLA, para quienes trabajé, no le tenían simpatía a mis manuscritos, mientras ellos prepa-

\footnotetext{
'Para una explicación más detallada del Proyecto Fronterizo de Investigación, patrocinado por la Fundación Carnegie en 1955, véase Loomis (1974), Loomis et al., (1966) Stoddard (1969) y Stoddard (1969, 1970).

${ }^{6}$ En la Universidad Estatal de Michigan coordiné más de una docena de investigaciones en esa rama para el Departamento de Economía Agrícola. En Desmoines, lowa, trabajé en un despacho legal como investigador externo, considerando la posibilidad de ir a la escuela de derecho. También, los dos años de impartir clases en una escuela de medicina y mi subsiguiente investigación con profesionales de la salud me ha otorgado algún entendimiento del área. Mi extensa rama de investigación me acercó a ótras.
} 
raban capítulos para libros y artículos para revistas profesionales de gran prestigio y visibilidad. Entonces me percaté de que yo tendría que publicar mis escritos por mí mismo; y asi lo hice.

Siguió mi búsqueda insaciable por más contactos y oportunidades multidisciplinarias, para intercambiar conceptos e información con colegas fuera de mi rama académica, con los costos económicos que representa la suscripción a revistas, folletos y membresías de organizaciónes y sin las recompensas de apoyo o aceptación de mis colegas entre mi rama académica principal (Stoddard y Mc Conville, 1978). Este es el núcleo de la comunidad multidisciplinaria. La convivencia con colegas académicos se reemplaza con contactos e intercambios con académicos de otras disciplinas, empujando al académico en general, a una posición marginada en su departamento o disciplina. Si desea seguir siendo aceptado en su especialidad, debe mantener su experiencia en esa área, por encima de sus intereses multidisciplinarios. $\mathrm{Si}$ debe conservar un grupo de referencia entre investigadores, deberá dedicar un buen tiempo y esfuerzo, en mantenerse en contacto en su propia rama y conceptos. Esta dualidad seguido causa tales presiones temporales y económicas al individuo, que lo hacen mediocre en cualquiera de las dos áreas, como resultado del rol ambivalente y de los recursos tan limitados.

Además de los anteriores colegas del proyecto fronterizo de Carnegie, empecé: aumentar mi lista de académicos interesados en la frontera. Algunas veces un becado visitante dejaría su nombre en tanto buscaba recursos materiales para elaborar su trabajo. Los manuscritos de periódicos y fundaciones tenían bibliografías y referencias de gente con quienes yo entraba en contacto. Seguido, candidatos a la maestría o doctorado venian o escribían solicitando información de "otros que tuviesen los mismos intereses". Mi lugar en la Universidad de Texas en El Paso, me colocó en una natural "caseta de información" de referencias bibliográficas, aún para el personal que estaba entrenado para estas tareas en los proyectos de investigación fronterizos. Algunos preguntaron sobre las "plantas gemelas", la migración mexicana o tendencias demográficas; sobre la literatura de los indios kickapoo en México, el comercio y las estadísticas económicas México-EE.UU., los autores que han utilizado determinadas perspectivas teóricas al escribir sobre la frontera, de las colonias mormonas y menonitas de México y acerca de otros temas o sub-culturas.

El siguiente paso para formalizar el campo de los estudios fronterizos ${ }^{8}$, se dirigió a ofrecer un criterio que distinguiera los estudios étnicos o académicos, haciendo investigación en la frontera, de aquellos que hicieran inves-

8El término territorios fronterizos (en plural) es un aspecto significativo para el verdadero en fogue multidisciplinario del estudio de la región. Existen tantas regiones fronterizas como hay disciplinas estudiándolas; desde la no existencia de una frontera para los arqueólogos, antropólogos y los primeros autores de historia, hasta una línea política legal que abarca el Golfo de México hasta California según se concibe por las ciencias sociales contemporáneas. 
tigación de los territorios fronterizos, conscientes de los efectos de la presercia de la frontera en sus análisis (Stoddard, 1974). También se incluyeron las listas actualizadas de los académicos de los territorios fronterizos y sus publicaciones, así como algunos patrocinadores potenciales de investigación. $E$ efecto catalítico que tuvo esta publicación en muchos profesores e investigedores me acarreó muchas cartas preguntando el porqué no los había inclue do, demostrando la necesidad de que existiera una sociedad con membresí abicrta de académicos con intereses en territorios fronterizos. Esta organización formal también serviría como vehículo para compartir información. ideas y posiblemente para apoyar una revista dedicado a la academia multidisciplinaria. La función de la "caseta" se necesitaba mucho, tanto como ma ticmpo para responder a las docenas de cartas que cada semana hacian preguntas. Más aún, debería existir para los colegas una colección de referencie de lo que sabemos de la frontera en nuestras respectivas disciplinas, y un plan para hacer un libro de referencia erudito que ayudara a los escolares en todas las disciplinas de la región. Muchas de estas inetas proyectadas se realizarían en los siguientes cinco o seis años.

En la junta de 1976 de la Asociación Occidental de Ciencias Sociales, un puĩado de académicos interesados en la frontera, se reunieron para validar la Asociación de Académicos del Territorio Fronterizo (Assuciation of Borderlands Scholars. A.B.S.). leyes y estructuras de organización que yo previamente disenić $\mathrm{c}$ hice circular. Mc pidieron que fuese presidente durante los años de organización y tratando de aumentar su atractivo, hice un esfuerzo por asistir a reuniones profesionales de arqueología, ciencias políticas, economía. historia, geografía y sociología: formando mesas multidisciplinarias y distribuyendo formas de membresia y objetivos de la A.B.S.

Casi al mismo tiempo un grupo de cinco académicos habían terminado una revisión sobre el listado de Iistudios sobre Territorios Fronterizos en sus disciplinas respectivis, que había sido publicado en la Revista de Ciencias Sociales (Stoddard, 1975) recién impresa ${ }^{9}$. La membresía de la ABS aumentó rápidamente a más de doscientos miembros, reflejando diversidad nacional y disciplinaria de cuatro países y más de veinticinco cstados de la Unión Americana, representando una docena de campos disciplinarios y académicos. $\mathrm{La}$ mayor concentración fuc en el área de geografia, cuando los miembros de la Conferencia de Géógrafos Latinoamericanistas (CLAG) se adhirieron en masa y abarcaron una tercera parte de la membresía total de la ABS. Sus miembros han dominado las posiciones de liderazgo en la organización debido a su número, visibilidad y sabida experiencia en investigación de territorios fronterizos. Esta notoria concentración en geografía se ha reflejado en las interre-

9Inicialmente formulado el papel de "documentos establecidos" para las reuniones de la Asociación Occidental de Ciencias Sociales, se aumentaron, editaron y se establecieron para las eventuales publicaciones de 1975/76 como resultado del simposio. 
laciones ale los miembros de la organización de geógrafos, así como en sus ideas, concoptos y métodos de investigación, dado que esta área académica por sí solia ha permeado las publicaciones más recientes sobre estudios fronterizos.

()tro problema único en proyectos que involucraban la coordinación de actividades de varias disciplinas, se manifestó en el simposio publicado. De los cinco artículos separados se formó una bibliografía general que sirviera para todos. Afortunadamente, la revista no impuso a su formato las citas bibliográficas tradicionales, puesto esto hubiera dificultado el trabajo, difícil de por sí de unificar los materiales de referencia de disciplinas que usan diferente forma.

La ABS publicó un folleto cada dos años, llamado lironteras ${ }^{10}$, que se apoyaba en las habilidades cartográficas de David Hornbeck (California State Northridge) y luego en las de Daniel Dillman (Northern Illinois). Al fin existiá un medio para intercambiar información entre los académicos interesados en temas fronterizos, aunque resultaba difícil actualizar los datos debido al intervalo que había entre cada folleto. No obstante, sirvió para reafirmar y afianzar las metas de la ABS en lo particular, y de los estudios fronterizos en general.

Parecia existir una creciente necesidad por un volumen de referencias ichtrales que reuniera las referencias de todas las disciplinas involucradas en lus estudios fronterizos. Puesto que la actual membresía de ABS contaba con imuchos expertos en áreas determinadas, o personas conocedoras de la literaIIIra en ciertos temas, se giraron invitaciones a participar. De entre los voluntarios se seleccionó a los individuos mejor calificados para los diferentes tullas y, a través de un arduo proceso de edición y revisión, los manuscritos finalmente se agruparon. Los intentos para obtener subsidios no se materializaron (Stoddard, 1981) pero por dedicación profesional de los contribuyentes, el Libro de Referencias de Territorios Fronterizos (Stoddard, et al., 1982) se hizo una realidad 11 . El volumen de 60 capítulos con similar núwero de temas, informaría a las agencias oficiales, académicos y público en general, respecto de cómo tener acceso a la información disponible. Un pro-

\footnotetext{
10 Este nombre se cambió luego al de Frontera, porque el uso plural fue reclamado por otras operaciones emergentes con orientación fronteriza. Pero, reflexionando más detenidamente, "la cultura fronteriza" compartida en dos regiones nacionales que están experimentando marginación en su país, probablemente refleja un punto de vista más acertado de cómo funciona la frontera, que como lo hace el término en plural que implica dos frontetas.

11 El Libro de Referencia de los Territorios Fronterizos, fue un sueño mío antes de 1970 . Iba a ser un libro de lecturas cubriendo varias instituciones de cultura fronteriza que claramente describiera cada una y que corrikiera las falsas impresiones existentes. A medida que se desarrollaba como un volumen enciclopédico con un contexto más amplio, la emoción de su potencial aportación final (mientras trabajaba con escolares dedicados que hicieron sus aportaciones sin compensación) opacó tanto las frustaciones y fracasos por encontrar subsidios como las pérdidas económicas personales (para una historia más completa, véase el caso de la historia en Stoddard, 1982b).
} 
yecto paralelo, el Atlas Fronterizo, no fue posible realizarlo sin los fondos $\mathrm{x}=$ queridos, como se había estimado en 1976, pero actualmente se lleva a cabo en California bajo el patrocinio de UC MEXUS.

Un proyecto acompañante al de Referencias de Territorios Fronterizo: fue el establecer un repositorio de material de consulta relevante en el ára de estudios fronterizos, especialmente algún material fugitivo 12 citado ex el Libro de Referencias. Usando mi propia colección de artículos no publicados, reportes de edición limitada y material relacionado con los estudios fromterizos, se inició la formación de un archivo en continuo crecimiento, haciézdose arreglos por escrito con la biblioteca de la Universidad de Texas en E Paso, con el fin de colocar ahí esta colección especial. El archivo creceria constantemente a medida que los miembros de la ABS mandaran sus libros copias, escritos no publicados o materiales de referencia. Desafortunadamente, la propuesta de aceptar toda la colección de publicaciones de territorios fronterizos en un solo lugar, no fue aceptada por la biblioteca de la UTEP: : la ubicación de la colección fue temporalmente pospuesta en los últimos meses de 1981. Pudiera ser que en fecha futura la ambicionada colección de archivo de la frontera logre estar disponible tal como se había planeado. Sir embargo, en la actualidad, muchos de los nuevos institutos y centros de investigación de territorios fronterizos, están tratando de acumular referencias para perpetuar sus objetivos y lograr la imagen de "caseta central" de dicho material. Esto representa una amenaza a la ABS como organización profesional, en varias formas. Primeramente, quita uno de los fines más significativos para la sociedad de profesionistas en esta área. En segundo término, proliferarán la localización de las "colecciones" de referencias en diversos centros $\mathbf{y}$ bibliotecas, lo que resultará en un aumento del costo que un académico tenga que cubrir para realizar una completa revisión del material. Por último, las políticas institucionales diferenciales afectarán la acumulación de materiales y la orientación hacia un campo determinado, más que la orientación multidisciplinaria de una sociedad de profesionistas. Eventualmente la necesidad de "reunir electrónicamente" puede reducir el espacio entre colecciones. pero se inclinará a ordenar los materiales dentro de las disciplinas académicas convencionales, más que como lo ha hecho la organización ABS.

Unos pocos han sido los centros que han tratado de extender sus acervos de referencias, entre los que se incluyen el Comité de Servicios de Amigos Americanos (American Friends Service Committee), cuyos folletos comenzaron en México al mismo tiempo que se formó la ABS. El boletín de noticias de UC MEXUS trata de hacer la función de "caseta central" para investigaciones en proceso, reuniones, conferencias. Del Centro Estatal de Recursos en

$12 \mathrm{Al}$ material fugitivo se le llama asf́ porque se publica en un formato no regular, normalmente sin información necesaria para una completa cita bibliográfica, y con tan pocas copias que no son facilitadas en los repositorios centrales. Documentos profesionales (exceptuando los extractos publicirios permanentemente en los resúmenes de los índices) caen en esta categoría aún cuando muchos resultan ser buenas aportaciones al conocimiento científico, pero que no logran sobrevivir como los artículos de periódico o capítulos de libro. 
el Area Fronteriza en San Diego se trasladó una pequeña colección a la librería de la Universidad, donde se ha ampliando merced a una búsqueda intensiva de material básico. El Centro de Estudios Chicanos en UCLA ha aceptado los materiales fronterizos en su amplio acervo, y pequeñas colecciones están esparcidas de Oriente a Occidente y de Nueva York a Texas ${ }^{13}$. Eventualmente, los jóvenes académicos interesados en investigaciones relacionadas con la frontera se acercarán a los centros en los que ya se llevan a cabo investigaciones y programas. Los que persiguen orientación general o cuyo interés persigue temas de poca popularidad actualmente lo deben hacer a su propio riesgo, sabiendo que los manuscritos o reportes que tienen un amplio terreno disciplinario son más difíciles para evaluarse, publicarse o usarse como base de solicitúdes para realizar investigación subsidiada (Stoddard, 1982b).

\section{AMPLIANDO PERSPECTIVAS: ¿RIESGO PROFESIONAL?}

$\mathrm{El}$ académico multidisciplinario puede tener al alcance de sus manos muchos más puntos de vista y conceptos para analizar un problema determinado. Es así que al acostumbrarse a consultar las disciplinas vecinas en busca de información sustantiva o marcos conceptuales, los intereses del académico pueden ser afectados. En muchas instancias los conceptos "prestados" se convierten en contaminantes de un tema dado. Puesto que la nueva aportación no se originó dentro del propio campo académico, hay una tendencía de negar aceptación de ideas entre los colegas del investigador. No sólo el pedir prestado legitimiza otras disciplinas vecinas como poseedores de la verdad, sino que la nueva perspectiva generalmente señala inconsistencias en el pensamiento tradicional o la verdad admitida entre los compañeros académicos. Esta no es una situación favorable para los académicos jóvenes que están tratando de obtener ocupación para subsistir, aconteciendo lo mismo con los académicos maduros que tratan de hacer aportaciones sustanciales. Extractos de mi experiencia ilustran este punto.

Al leer unos artículos de historia y antropologia me encontré con una descripción de la antigua sociedad griega en la que el rol de ciudadano confería acceso a la plena participación democrática, mientras que los no ciudadanos (la mitad de la población) no tenían tales derechos. Como liberal practicante, para mi fue una sorpresa descubrir que estábamos creando un sistema americano que le permitía a cada individuo de nuestra nación esos dere-

\footnotetext{
13Sólo algunos de estos incluyen SUNY-Buffalo, los Departamentos de Antropología de Cornell y SMU, el Museo del Desierta de Tucson, la Biblioteca Huntington de Califotnia, las pequeñas colecciones en los museas de arte de Dallas y Santa Fe, y los dísgregados institutos sobre América Latina, como la Universidad de WisconsinMilwakee, Tulane, Universidad de Nuevo México y otras. Los colectores más recientes como el Centro Estatal de California Fullerton, el Colegio de México sucursal de Tijuana, el Centro de Investigación en Texas Southmost y otras, muestran la rảpida fragmentación de recursos en la rama de territorios fronterizos.
} 
chos gozados únicamente por la élite de Grecia (Stoddard, 1967). De manera parecida, "la etapa de oro del hombre" tan nostálgicamente descrita por Rosseau, fue formulada desde los tempranos contactos de los europeos con los nativos de América. Los tainos de Española y Massachusetts eran grupos poco educados, que se suponía eran ejemplos embriónicos del hombre antes de la civilización, No solamente la antropología legitimó su cultura como "taino contemporáneo" más que "caucasoide primitivo"; sin embargo, otras culturas indígenas tales como los comanche, los kowa y los yaquis, no nos muestran docilidad y cualidades duraderas de los sedentarios tainos, pues no entran en la teoría de la humanidad primitiva como dice Rosseau. Esta concepción, más que de un pasado noble, provenía de las amargas descripciones de los acontecimiento consignados en los diarios de los conquistadores. Unicamente utilizando materiales de referencia académicos provenientes de disciplinas distintas a la filosofía o las ciencias sociales teóricas, podrían estos esquemas pseudo-cient íficos cuestionarse y considerarse deficientes.

Estıdos Undos de América, después de la II Guerra Mundial, hablaba de ayudar a las "naciones subdesarrolladas", etiqueta que colocaba a las naciones no industrializadas en una posición inferior. El modclo evolucionista del desarrollo de la sociedad, elaborado por el abogado-antropólogo Henry Morgan, colocó estas naciones en un temprano período de desarrollo. Asumiendo que los avances tecnológicos de la cultura reflejaban su nivel social y moral, permitía a las naciones de mayores avances tecnológicos verse como el producto acabado del inevitable proceso evolutivo de la humanidad. De igual manera, las minorías étnicas de los Estados Unidos fueron clasificadas como "privados culturalmente", porque su origen de clase baja no reflejaba los valores de la clase media norteamericana. Solamente cuando se emplea cl concepto de relatividad cultural (Herskovits, 1948) las culturas indigenas se hacen contemporáneas de nosotros, y nuestras minorías étnicas se consideran diferentes culturalmente. Otras aberraciones se han impuesto en lo que respecta a la gente méxico-americana del suroeste $y$, aunque es triste admitirlo, se ha debido a la labor de los "científicos sociales". Dõs desafortunados estereotipos serán suficientes para ilustrar estos obstáculos pseudo-científicos.

Una falacia histórica se ha desarrollado al describir los antiguos imperios de México y Centroamérica, al considerar que los aztecas estaban en el cenit de la creatividad y tradición cultural del valor y honor ${ }^{14}$. Desde una perspectiva más objetiva, se puede ubservar que la contribución hecha a

14 Véase Stoddard (1978c: 35.41) para un recuento de esta mala representación. Tanibién. Cumberland (1968) sabiamente anota el genio para organizar y hacer tratos en vez de la creatividad cultural como la fuerza inherente al imperio azteca. 
La cultura mexicana durante 125 años por parte de los aztecas se debe más 2] hecho accidental de estar en el poder al arribo de los historiadores europeos, en comparación a las contribuciones hechas por los mayas, olmecas, zapotecas y toltecas.

Los aztecas, al dejar de ser gente que pagaba tributo durante el reinado de Itzcoatl, la élite política trató de glorificar un pasado brillante destruyendo sus historias y creando mitologías de ellas, y lo lograron (León Portilla, 1960:11). Esta narrativa fabricada, utilizada por la mayoría de los historiadores, desde Prescott ${ }^{15}$ hasta los escritores contemporáneos, fue empleada después como la cosmología chicana (Rendón, 1971).

La falacia asociada con las configuraciones culturales relegaba "los valores de orientaciones mexico-americanos" a una posición y comportamiento indeseable, mientras se prefería el conjunto de "orientaciones angloumeric anos". Estudios de problemas de salud méxico-americanos hechos por Saunders (1954), Clark (1959), Rubel (1966) y muchos otros, aceptaron éstas como verdaderas diferenciàs étnicas. A partir de múltiples muestras rurales y urbanas se puede observar inmediatamente que la orientación "étnica" de los méxico-americanos refleja los valores de la clase trabajadora, mientras que los valores de los "anglo-americanos", son el núcleo de la clase media estadounidense. (Stoddard, 1973: 38-51). Un concepto muy parecido, al de cultura folk aplicado a los habitantes de los barrios marginales (Kiev, 1968), fue incorrectamente considerado como caracter ística de los mexico-americanos rurales ${ }^{16}$. De la antropología surge una explicación más sofisticada de las culturas folk y campesina, explicación que corrige estas equívocas impresiones (véase Foster, 1953: Leeds, 1968) y aclara las limitaciones de estos conceptos que han pasado a nosotros en forma escrita. Pero aún el académico multidisciplinario desarrolla cierta miopía escolástica, según revela mi experiencia.

Desde mis primeros años en la universidad, mi especialización en la cultura mexico-americana excluyó la extensa investigación necesaria para familiarizarme con muchas de las culturas indígenas. Aunque yo conocía en lo general las diferencias de las gentes en la llanura y en los pueblos, ignoraba las contribuciones de otros grupos raciales y étnicos a la rica herencia del Suroeste. Ahora, por más de una década, mi orientación multidisciplinaria me ha

\footnotetext{
15Mientras que la Conquista de México, de Willam H. Prescott (1936) es considerada comúnmente como la historia clásica de la invasión española, pocos historiadores están familiarizados con el origen de este trabajo. Trabajando bajo influencias negativas y limitadas para escuchar sólo las narraciones de los primeros exploradores españoles, Prescott dificilmente creó una novela nartativa como se la ímaginó, incluyendo descripéiones del emplerador Moctezuma, del comedor y sus adornos de oro, los utensilios usados en honrar a Cortés y sus hombres. Ios cuales no aparecen en las reseñas. (Véase Humphreys, 1959).

16 Investigaciones más recientes (véase Nall y Spielberg. 1967 por ejemplo) muestran que la conducta extrovertida de los practicantes del folk, su disponibilidad y precios bajos atraen al imexico-americano de bajos in. gresos, más que la posible atracción ejercida por los valores de orientación.
} 
forzado a extender mis conocimientos de los grupos negros y orientales. cuya presencia tuvo un fuerte impacto en nuestra cultura actual. En los libros de historia del Suroeste está ausente la narración de las heroicas jornadas de los regimientos de caballería e infantería $9{ }^{\circ}, 19^{\circ}, 24^{\circ}$ y $25^{\circ}$, integrados en su totalidad por negros, mayoritariamente responsables de la construcción y mantenimiento de los fuertes militares en la inhóspita expansión de esta se mi-árida región. Además, las contribuciones de los vaqueros negros al desarrollo de la industria ganadera en Texas no puede ser minimizada. Nuestro trato inhumano a los orientales y su presencia, nos recuerdan constantemente que la "Libertad para todos" ha sido un ideal verbal, no una política legal para aquellos racialmente diferentes por su ascendencia china o japonesa ${ }^{37}$

Los estudiantes universitarios que dedicados a la lengua y literatura espart:las tienden a recibir una imagen gloriosa de la cultura hispana, dándole ux alto valor al arte del renacimiento del siglo XVI, llamado "Siglo de Oro". en la que la arquitectura del infinito detalle y el adorno con hoja de oro sobresale como una contribución al espíritu indomable del hombre. El arte * escultura de esa época también son aclamados como el más noble de los ez fuerzos del hombre para crear belleza. Estas nociones románticas pueden modificarse desde el punto de vista multidisciplinario más amplio , que cuestiona la manera que los artesanos españoles fueron abastecidos, cómo sostuvieron los trabajadores necesarios y obtuvieron recursos financieros para realizasus creaciones artísticas. Obviamente un sobrante monetario estaba dispuezto para apoyar las actividades de sobrevivencia no-básica, que a simple vita parecen ser resultado de la suerte y de la excelencia aventurera. Pero ur escrutinio más detenido muestra que las riquezas que permitieron la expansión del arte en la península española, se pagaron por medio del sufrimiento humano en el Nuevo Mundo; la recién descubierta fuente de riquezas. Durante los primeros 125 años de la conquista, el número de indios que moríacada sesenta días era igual al número de españoles que se iban al Nuevo Mundo. para mandar las riquezas a su tierra. Es así que desde esta perspectiva más amplia, se ve el ocio disfrutado en la Península Española como resultado de la explotación inhumana a la que se sometió a las gentes indígenas de América (Cumberland, 1968:43).

También, dentro de la década pasada, he empezado a incorporar algunos de mis conocimientos arqueológicos a los reportes "contemporáneos". Por ejemplo, un reporte de investigación que analiza el incidente de la "cortina de tortilla", se enfoca a partir de las opiniones de los líderes de Ciudad Juá-

\footnotetext{
17 Capítulos separados sobre las aportaciones de las culturas negro oriental y del Medio Oriente, fueron includos en el Libro de Referencias sobre Territorios Eronterizos escrito por mi, no tanto por mis investigacione: y experiencia profunda, sino porque ningún escolar tomaba en consideración estas contribuciones culturales tradicionales. Sin estos capítulos El Libro de Referencias hubiera creado y legitimado el estereotipo de que dichas culturas no hicieron aportaciones, al igual que casi el 100 por ciento de los textos hoy en uso en las escuelas del Suroeste.
} 
rez y EI Paso, respecto de las políticas de contención en la frontera implementadas por el Servicio de Inmigración y Naturalización (Stoddard, et al., 1978). Pero, pongamos la narrativa en su perspectiva propia: el Suroeste con sus lozanas tierras húmedas, pantanosas y reforzadas por las lluvias de los monzones en cuya vegetación semitropical vivían los mamuts, muestra la necesidad humana de adaptarse de una condición semi-tropical a una semiárida, tanto como los problemas políticos contemporáneos de origen binacional. De igual manera, cuando me pedían dar una descripción enciclopédica de la "cultura fronteriza" (Meier and Rivera, 1981: 4750), inclui el antecedente pre-histórico como precursor necesario para interpretar las instituciones históricas y modernas.

Para eliminar las nociones etnocéntricas de los planificadores urbanos, que declaran haber empezado como profesión en este siglo, basta un breve vistazo a Pueblo Bonita, situado en el Cañón Chaco al norte de Nuevo México, entre 1000 y 1200 D.C., para darse cuenta que las gentes de este hemisferio no son los "indios tontos", esterotipo representado en la televisión, películas y en nuestros distorsionados libros de historia. Asimismo, los 30,000 habitantes de Cahokia18, situada cerca de la actual ciudad de Kansas, es ignorada cuando los historiadores hablan de las avanzadas culturas mayas y aztecas, aún cuando Cahokia estuvo en su plenitud tres siglos antes de la conquista española a los aztecas. No todas las culturas de importancia se localizaban en Perú, Guatemala y México. De la misma forma, no todas las culturas indígenas eran tribus nómadas exóticas.

Los científicos sociales contemporáneos de vez en cuando desarrollan nuevas y más sanas ideas al abordar un sólo problema desde más de una perspectiva académica. Por ejemplo, los economistas que aprenden algo de la psicología social del "sentirse rico", son capaces de interpretar más acertadamente las reacciones económicas de la gente que aquellos que sólo lo interpretan a partir del valor del dólar. El considerar la carencia de dólar como una condición de pobreza, es ignorar las aportaciones críticas socio-psicológicas respecto de las percepciones humanas. Dependiendo de los patrones (actuales o anteriormente obtenidos en un pais extranjero) que se usen para determinar el nivel de pobreza -llamado privación relativa-lo que para una persona con ingreso inferior al salario mínimo puede ser la abundancia, sería la pobreza para otro, según se refleja en estudios empíricos de extranjeros ilegales mexicanos (Stoddard, 1976a, 1976b, 1978a: 52-64, 1979b). La naturaleza institucional de la migración mexicana también refuta la simplista noción legal de que el movimiento tradicional a través de la frontera internacional en violación de un mosaico de estatutos de inmigración contradictorias, es el equivalente a un intento criminal.

18 Además de Cahokia, las excavaciones hechas en el rancho Koster cerca de Dampsville, Illinois, al inicio de 1970, han descubierto un lugar, en un fértil valle, que contiene muy diferentes culturas con una antigüedad de seís a siete mil años o más. La precisión de las excavaciones y la entereza de sus investigadores, nos da cuenta de un desarrollo cultural en el norte de América equivalente al valle de Tehuacán en México o del Tikal maya. (Véase Roper, 1979; Struever and Holton, 1979). 
De la misma manera, la diplomacia internaciona admite diferentes grados de solución a problemas, cuando se miden los intreses nacionales contra las consecuencias regionales o locales. Aunque los statutos federales sean creados en Washington D.C. y México, D.F. para sus respectivas jurisdicciones fronterizas, las ciudades e instituciones locales sol:mente pueden subsistir si ignoran, enredan o traducen los mandatos centrdes, en procedimientos aceptables para los locales (Stoddard, 1979a, 1982a. Cuando los expertos de una distinguida institución de enseñanza superior di Noroeste o del Oeste llegan a la frontera, listos para resolver los problemasutilizando reportes formales de información y sus modelos sacados de los estudios hechos de ciudades del interior, seguido experimentan el fracaso. Esto no es debido a falta de sofisticación, sino consecuencia de su incapacidad para descifrar cómo funcionan las redes de conexiones informales, y cómo los reportes se adecúan para satisfacer las espectativas de los burócratas fronterizos. La claridad ganada con el uso del sistema informal de análisis, es útil para esas disciplinas que tienden a analizar datos y estadisticas formales.

En resumen, la clara articulación de los problemas fronterizos y sus con. diciones a través de las variadas perspectivas de esfuerzos multidisciplinarios proporciona muchos conocimientos que las investigaciones estrictamente disciplinarias no generan. Pero es igualmente claro que la coordinación y acep. tación de los académicos de orientación unidisciplinaria en un más amplia contexto multidisciplinario es una amenaza, tanto para las disciplinas repre. sentadas, como para la conservación de las creencias que son básicas para cadu rama académica. Así, los esfuerzos multidisciplinarios que provengan de 1 il acadérnico solo o de un equipo de colegas, enfrentan peligros similares.

\section{TENDENCIAS Y DILEMAS PRESENTES PARA LA INVESTIGACION FRONTFRIZA MULTIDISCIPI.INARIA}

Así como hemos visto que un simple asunto político ha logrado cambize los patroncs de votación y las estrategias de campaña para puestos públicos hemos igualmente observado cómo la multiplicación de fondos para investrgación y publicación de temas sensacionalistas (como los emigrados ilegales - las maquiladoras en comunidades de la frontera mexicana) lan creado una nueva entidad parasitaria conocida como los "bandidos del cinto" de Washing ton, D.C. 19. Estos expertos instantáneos en problemas fronterizos han reunido teportes, copias y publicaciones de académicos respetables en estudios fronterizos, y las han usado con éxito para obtener becas de agencias federales y estatales que no sospechan de ellos. Pero los márgenes de ganancias para este tipo de investigación, en este tiempo de crisis económica, seguramenté

\footnotetext{
19 "Bandidos del cinto" es el término poco agraciado que se ha dado a las recién formadas organizaciones de asesoría e investigación ubicadas en las afueras de la ciudad, alrededor de Washington, D.C., donde sus lujosos apartamentos pueden verse desde la carretera. Probablemente su renta y su papeleria membretada sean las inversiones más visibles hechas por estas organizaciones, que viven de becas obtenidas en las agencias y fundaciones de Washington, D) C.
} 
darán cuenta de estos oportunistas. Desafortunadamente, los legítimos académicos fronterizos no son conocidos en el enrarecido ambiente de Washington y tendrán que obtener sus fondos con el mismo esfuerzo del pasado, pero en un contexto en el que se dispone de menos fondos para la investigación.

En el pasado, más investigadores fronterizas y planes de desarrollo han sido subsidiados por las agencias y programas del gobierno mexicano. Parecida a la situación de los Estados Unidos, los subsidios están muy allegados a los temas políticos y populares que se enfocan a partir de los modelos de las teorías de la dependencia y de los marcos teóricos neo-marxistas 20. Debido a la falta de académicos mexicanos entrenados en investigación, las oportunidades de colaboración entre los investigadores de EE.UU. y México, se reducen a pocos individuos, y éstos tienen tantas presentaciones y conferencias que les quedan pocos recursos para participar en el proyecto de investigación mismo. Debido a los problemas económicos actuales de México, la investigación en colaboración sufrirá traumas antes de que el subsidio económico ya designado se haga efectivo. Parece que la investigación en colaboración será "pospuesta" para que pocos proyectos nuevos se inicien, mientras que los antiguos tendrán que esperar para llevarse a cabo.

Mientras que en los primeros años de la década de 1970 pocos académicos se esforzaban para trabajar en la investigación fronteriza con magros recursos, en los últimos años ha habido cambios. Los que antes eran pequeños grupos de investigación en la Pan American University, Trinity, University of Texas at El Paso, Arizona y San Diego State, son opacados con programas en los sistemas universitarios de California y Texas. El programa de la UC MEXUS va aumentando en visibilidad. El Centro de San Diego de la Universidad de California, ha sido como el frijol de Juanito, un fenómeno que creció la noche a la mañana, junto con su conexión de Tijuana, relacionada con el Colegio de México. La Universidad de Stanford, la Universidad de Texas en Austin, la Universidad Duke y una o dos más, parecen ser las grandes ganadoras en la "rifa de estudios fronterizos". Un gran peligro para los estudios de la frontera es que mientras estos centros persiguen reconocimiento y dedican mayores esfuerzos para definir su campo de acción, proporcionar publicaciones y distribuir materiales de investigación, la función anteriormente desempeñada por la Asociación de Académicos de Territorios Fronterizos (ABS) puede ser supeditada a los intereses de una universidad o a la de los sistemas de universidades. La lealtad de los profesionales de la investigación

\footnotetext{
20 Las explicaciones estructurales de los problemas fronterizos que atañen al contraste entre el rico Estados Unidos y el relativamente más pobre México, en el borde de la frontera, es un tema popular entre los escolares mexicanos contemporáneos. Sin embargo, a la teoria de la dependencia que se usa para explicar el rol del anglo-americano como causa de la mayoría de las miserias de México, le falta substancia teónca y práctica para considerarla como el agente monocausal (véase Bath y James, 1976). El caso reciente de la devaluación del peso mexicano sugiere que más que incongruencias estructurales con los EE.UU., la naturaleza del sistemático saqueo sexenal de la hacienda mexicana por los presiedentes salientes y sus amigos, es un más serio (y corregible) problema que las arengas referidas a las maquiladoras pertenecientes a corporaciones multinacionales ubicadas en la frontera.
} 
tiende a orientarse hacia las fuentes de recursos para investigación disponibles y la fragmentación de las funciones profesionales que antes había ejecutado la Asociación de Estudios Fronterizos se transforma en intereses más específicos, en temas relacionados con frontera por parte de una solo Universidad o del sistema universitario, los cuales podrían debilitar los fundamentos profesionales, tanto como las orientaciones multidisiplinarias de los estudios de territorios fronterizos.

Los jóvenes principiantes harian bien en deshacerse del romanticismo de la colaboración multidisciplinaria, a menos que las oportunidades de subsidios sean buenas y garantizadas. Para el académico bien establecido, especialmente uno con publicaciones y renombre en su ramo académico, representa un riesgo mínimo el involucrarse en esfuerzos multidisciplinarios que no resulten en artículos o monografías publicados por los periódicos de la prestigiosa prensa. Pero en algún punto, los académicos más antiguos y establecidos deben ser reemplazados por esos que están ahora en entrenamiento, y si las actuales tendencias continúan en las que las oportunidades de investigación se ofrecen en temas específicos o en temas de una rama académica, la naturaleza multidisciplinaria de estudios de territorios fronterizos disminuirá y terminará. Aun en los mejores tiempos, el anonimato en la carrera es uno de los pocos resultados garantizados para quien se vuelve un escolar multidisciplinario. Y dado que actualmente no son los mejores tiempos para investigación general, el cíclico capricho de la sensibilidad del público respecto a los problemas específicos de la frontera, puede representar el mayor peligro para las investigaciones profesionales y multidisciplinarias en los estudios de territorios fronterizos.

\section{BJBLIOGRAFIA}

BATH, C. Richard and Dilmus D. James. Dependency Analysis of Latin America: Some Criticisms, SSuggestions. Latin American Research Review 11 (Fall): 1976 pp. 3-54.

BENEDICT, Ruth. Patterns of Culture. Houghton Mifflin N.Y. 1934.

CLARK, Margaret, Health in the Mexican-American Culture: A Community Study. University of Californs Press, Berkeley, 1959.

COLLIER, John. Indians of the Americas. The Long Hope. New American Library (Mentor) N.Y., 1947.

CUMBER LAND, Charles C. México: The Struggle for Modemity. Oxford University Press, N.Y., 1968.

FOSTER, George M. What is Folk Culture? American Anthropologist 55 (No. 2) 1953, pp. 159-173.

HANKE, Lewis. Aristotle and the American Indians: A Study in Race Prejudice in the Modern World Regnery Co., Chicago, 1959.

HERSKOVITS, Melville J-Man and His Works: The Science of Cultural Anthropology, Alfred A. Knopf, S, : 1948.

HUMPHREYS, R.A. William Hinkley Prescott: The Man and the Historian, Hispanic American Historia Review 39 (February), 1959, Pp. 1-19.

KEEN, Benjamín. The Black Legend Revisited: Assumptions and Realities, Hispanic American Historie Review 49 (November), 1969, pp, 703-719.

KIEV, Ari. Curanderismo: Mexican-American Folk Psychiatry. The Free Press, N,Y., 1968.

KLUCKHOHN, Florence Rockwood and Fred L. Strodtbeck. Variations in Value Orientations. Row, Peters $s \pi$ and Co., N.Y., 1961.

LAS CASAS, Bartolomé de. La Brevisima Relación de la Destrucción de las Indias in: John Francis Banncr (ed) Indian Labor in the Spanish Indies. D. C. Heath, Boston, 1966, Pp. 36-39 
LATTIMORE, Owen. Ordeal by Slander. Bantam Books, N.Y., 1951.

LEEDS, Anthony. The Anthropology of Cities: Some Methodological Issues. Institute of Latin American Studies. Offprint Series No. 80, Austin, 1968.

LEON PORTLLA, Miguel. The Concept of the State among the Ancient Aztecs. Alpha Kappa Deltan 30, 1960, pp. 7-13.

LOOMIS, Charles P. History and Results of the Michigan State University Cannegie Corporation Border Project. Paper presented to Southwestern Sociological Association (March), Dallas, 1974.

LOOMIS, Charles P., Zona K. Loomis, and Jeanne E. Gullahorn. Linkages of Mexico and the United States. East Lansing. Michigan State University Experiment Station Bulletin No. 14, 1966.

MEAD, Margaret. The Spanish Americans of New Mexico, U.S.A. in: Margaret Mead (ed), Cultural Patterns and Technological Chartge, UNESCO: Mentor Ed., 1955, pp, 151-177.

MEIER, Matt S. and Feliciano Rivera. Dictionary of Mexican American History. Greenwood Press, Wrstport. 1981.

NALL. Frank C. II and Joseph Speilberg. Social and Cultural Factor in Responses of Mexican-Americans to Medical Teatment. Journal of Health and Social Behavior 8 (December), 1967, pp. 299-308.

PRESCOTT, William H.History of the Conquest of Mexico. Modern Library, N. Y, 1936.

RENDON, Armando. Chicano Marifesto, The MacMillan Company, N. Y. 1971.

RUBEL. Arthut J. Across the Tracks: Mexican-Americans in a Texas City. University of Texas Press, Austin, 1966.

ROPER, Donna C. Archaeological Survey and Settlement Pattems in Central Mlinois. Illinois State Museum Scientific Papers Vol, XVI, 1979.

SAUNDERS, Lyle. Cultural Differences and Medical Care. Rusell Sage Foundation, N. Y.s 1954.

SMITH, T. Lynn. Values Held by People in Latin America Which Affect Technical Cooperation. Rural Sociol. ogy 21 (March), 1956, pp. 68-75.

STODDARD, Ellwyn R. Freedom Types und Ethnocentric Bias in the Liberal Tradition. Rocky Mountain Social Science Journal 4 (October), 1967, pp, 22-31.

- The U.S. Mexican Border A comparative Research Laboratory. Journal of Inter-American Studies 11 (July), 1969, pp. 477-488.

- Comparative Structures and Attitudes along the U.S. México Border in: E. R. Stoddard (ed) Comparative U. S. México Border Studies, Border-State University Consortium for Latin American, Occasional Paper No. 1, El Paso, 1970, pp, 1-38.

- Mexican Americans. Random Housc, N. Y., 1973.

- U, S. México Borderlands Studies: An Inventory of Scholars, Appraisal of Funding Resoutces and Research Prospects. University of Texas at El Paso Center for Inter-American Studies. El Paso, 1974.

(Ed) The Status of U.S. -Mexico Borderlands Studies. A.Multi-Disciplinary Symposium. Social Science Journal $12 / 13$ (October 1975/January 1976), 1975, pp. 1-112.

- Illegal Mexican Labor in the Borderlands: Institutionalized Support of an Unlawful Practice. Pacific Sociological Review 19 (April), 1976a, pp. 175-210.

- Conceptual Analysis of the "Aliena Invasion: Institutionalized Support of Illegal Mexican Aliens in the U.S. International Migration Review 10 (Summer), 1976b, pp. 157-189.

- Pattems of Poverty along the U.S. Mexico Border. University of Texas at El Paso Center for InterAmerican Studies, and Organization of U.S. Border Cities, El Paso, 1978a.

- Om "Buying and Selling Americu". An Ethnohistorical Approach to Territorial Disputes in the South. west. Paper presented to RMSSA, April 1973, in: E. R. Stoddard (ed) Two Unpublished Papers on South-

west. Paper presented to RMSSA, April 1973, in: E. R. Stoddard (ed) Two Linpublished Dapers on Southwest Ethnohistory. University of Texas at El Paso, 1978c.

- A 3-D Perspective of Water Gate Rip-offs along the Rio Grande: Water Allocations by Dam, District. In Will C. Kennedy (Ed) Environmental Problems along the Border, Border-State University Consortium for Latin America Occasional Paper No. 7, San Diego, 1979a, pp, 38-71.

- Border and Non-Border Illegal Mexican Aliensi A Commentary. Business Review 16 (My). El Paso, 1979b, PP. 21-27.

- Local and Regional Incongruities in Bi-National Diplomacy: Policy for the 1,S. Mexico Border. Policy Perspectives 2 (No. 1), 1982a, pp. 111-136.

- Multidisciplinary Research Funding A "Catch 22" Enigma, The American Sociologist 17 (November). $1982 \mathrm{~b}$.

STODDARD. Ellwyn R.. Oscar J. Martinez and Miguel A. Martínez Lasso, El Paso-Ciudad Juárez Relations and the "Tortilla Gurtain". A study of Local Adaptation to tederal Border Policies. El Paso Council on the Arts and Humanities, and the University of Texas at El Paso, El Paso, 1979.

STODDARD, Ellwyn R. and Lawrence McConville. The Effectiveness of Sociology in Training and Rewarding Scholar in a Multidisciplinary/Multicultural Field. Wstern Sociological Review 9 (Fall), 1978, pp.67-75.

STODDARD, Ellwyn R., Richard L. Nostrand and Jonathan P. West. (Eds) Borderlands Sourceboole. Norman: University of Oklahoma Press, 1982.

STRUEVER, Stuart and Felicia Antonelli Holton. Koster: Americans in Search of Their Prehistoric Past. Anchor Press, Garden City, 1979 